\title{
Raman Spectroscopy of Co-Doped ZnO Bulk Crystals
}

\author{
W. Szuszkiewicz ${ }^{a, *}$, J.F. Morhange ${ }^{b}$, Z. Golacki ${ }^{a}$, \\ A. Łusakowski ${ }^{a}$, M. Schumm ${ }^{c}$ And J. Geurts ${ }^{c}$ \\ ${ }^{a}$ Institute of Physics, Polish Academy of Sciences \\ al. Lotników 32/46, 02-668 Warszawa, Poland \\ ${ }^{b}$ Institut des Nanosciences de Paris, UPMC \\ 140 rue de Lourmel, 75015 Paris, France \\ ${ }^{c}$ Physikalisches Institut der Universität Würzburg \\ Am Hubland, 97074 Würzburg, Germany
}

Low-temperature Raman spectra of a bulk mixed crystal $\mathrm{Zn}_{1-x} \mathrm{Co}_{x} \mathrm{O}$ $(x=0.016)$ are shown and analyzed. Besides the common modes of the $\mathrm{ZnO}$ host lattice, electronic transitions related to the presence of $\mathrm{Co}^{2+}$ ions at the $\mathrm{Zn}$ sites are observed. In spite of the low Co concentration the presence of collective excitations corresponding to $\mathrm{Co}$ and $\mathrm{CoO}$ precipitates is also revealed.

PACS numbers: 75.50.Pp, 78.30.Fs

\section{Introduction and motivation}

In the recent few years, diluted magnetic semiconductors (DMS) have attracted once more a broad interest for their potential applications in future spin-based electronics and optoelectronics (spintronics) [1]. Among these materials, DMS grown on the basis of $\mathrm{ZnO}$ play a prominent role since room temperature ferromagnetism (RTFM) arising from carrier-mediated exchange interactions has been predicted for several transition metal (TM) doped $\mathrm{ZnO}$ including the $\mathrm{Zn}_{1-x} \mathrm{Co}_{x} \mathrm{O}$ mixed crystal $[2,3]$.

The determination of the magnetic properties of $\mathrm{Zn}_{1-x} \mathrm{Co}_{x} \mathrm{O}$ has been the object of several studies performed until now. The results of these studies were often ambiguous or even contradictory. This was due to the fact that the magnetic properties of this material depend on the conditions during the crystal growth, on the sample type (bulk single crystal, ceramic sample, thin layer, nanocrystal, or

*corresponding author; e-mail: szusz@ifpan.edu.pl 
nanowire), and on the after-growth treatment (e.g. annealing). In the case of polycrystalline bulk $\mathrm{Zn}_{1-x} \mathrm{Co}_{x} \mathrm{O}$ samples prepared by solid state reaction method, most of them exhibited a paramagnetic or even antiferromagnetic behavior at room temperature (RT). The RTFM observed in some $\mathrm{Zn}_{1-x} \mathrm{Co}_{x} \mathrm{O}$ samples was typically attributed either to the generation of oxygen vacancies or to the appearance of Co clusters [4, 5]. Similar explanations (or the formation of secondary phases containing $\mathrm{Co}$ ) were proposed for bulk polycrystalline ferromagnetic samples prepared by other methods. Experimental reports of RTFM in selected $\mathrm{Zn}_{1-x} \mathrm{Co}_{x} \mathrm{O}$ films (for details see, e.g., [6]) have further intensified the interest in this system. A key question for the understanding of all above-mentioned results is whether the material under consideration contains only randomly distributed TM ions at the substitutional position in the host crystal lattice or if the material contains also secondary phases like Co-related clusters or precipitates that are responsible for the observed magnetic properties.

A possible experimental method to study the structure properties of materials is the Raman spectroscopy. The Raman scattering studies on $\mathrm{Zn}_{1-x} \mathrm{Co}_{x} \mathrm{O}$ system started very recently, most of the measurements were performed at RT only. It is widely believed that the secondary crystal phases can be expected for $\mathrm{ZnO}$ containing at least $5 \%$ of Co. The presence of such precipitates as Co or $\mathrm{CoO}$ has been found in bulk samples $(x=0.05[7])$, various cobalt oxides or cobalt spinel have been found or suggested in selected mixed crystal layers $(x>0.05[8])$. Several local vibrational modes (LVMs) corresponding to sharp structures in the Raman spectra are expected for a TM impurity occupying a substitutional site in the crystal lattice (see, e.g., [9, 10]).

The goal of the present work is to get information about the position of the Co ions in the $\mathrm{ZnO}$ crystal lattice and about the possible presence of Co-related precipitates even in the low-composition mixed crystal range.

\section{Experimental}

For the growth of the $\mathrm{Zn}_{1-x} \mathrm{Co}_{x} \mathrm{O}$ crystals the chemical vapor transport (CVT) method was applied. The crystals were grown at the Institute of Physics, PAS, in Warsaw at about $1050-1100^{\circ} \mathrm{C}$ in Zn-rich conditions. Both inert $\left(\mathrm{Ar}, \mathrm{N}_{2}\right)$ and reactive $(\mathrm{Br}, \mathrm{Cl})$ gases were used in the growth system (in particular, the presence of $\mathrm{Br}$ or $\mathrm{Cl}$ supports the transport of dopants). Pure $\mathrm{ZnO}$ crystals were grown both in Zn-rich and stoichiometric conditions. The bulk samples containing approximately $1.6 \%$ of Co [11] have been selected and compared to a pure ZnO.

Typical Raman scattering measurements were performed using a Jobin-Yvon U1000 double monochromator in the spectral range from 0 to $650 \mathrm{~cm}^{-1}$ with a spectral resolution close to $1 \mathrm{~cm}^{-1}$. The $\mathrm{Ar}^{+}$laser lines with wavelengths $514.5 \mathrm{~nm}$ or $488 \mathrm{~nm}$ were applied for the excitation. A photon counting system and a S20 photomultiplier served as the detector system. For the low-temperature measurements the sample was placed on the cold finger of a continuous-flow helium cryostat. 


\section{Results and discussion}

Figure 1 shows a Raman spectrum taken at $T=15 \mathrm{~K}$ on a Co-doped $\mathrm{ZnO}$ sample in a wide spectral range. Beside the structures corresponding to typical and well-known host lattice modes (namely to $A_{1}$ (TO) $378 \mathrm{~cm}^{-1}, E_{1}$ (TO) $410 \mathrm{~cm}^{-1}$, and $E_{2}$ (high) $438 \mathrm{~cm}^{-1}$, respectively), several new peaks appeared in the spectrum. The structure at $134 \mathrm{~cm}^{-1}$ is due to scattering on $E_{2 \mathrm{~g}}$ phonon of hcp-Co [12], peaks at about $146 \mathrm{~cm}^{-1}$ and $300 \mathrm{~cm}^{-1}$ are related to CoO magnons [13, 14]. The temperature dependence of the Raman spectra (not shown here) confirmed these findings. The presence of such precipitates was reported previously for a $\mathrm{Zn}_{0.95} \mathrm{Co}_{0.05} \mathrm{O}$ sample [7]. The present data demonstrate that the secondary phases as the above-mentioned ones may be created even for a Co content much lower than that previously claimed as a limit for a one-phase $\mathrm{Zn}_{1-x} \mathrm{Co}_{x} \mathrm{O}$ mixed crystal. One should mention that in the case of nanocrystals such limit is at least as high as $x=0.1$ [15]. Probably the limit for the presence of precipitates depends on the crystal dimensionality and the observed trend could be explained by a "purification mechanism" related to the migration of Co towards the nearest crystal surface, but this effect requires further more detailed studies.

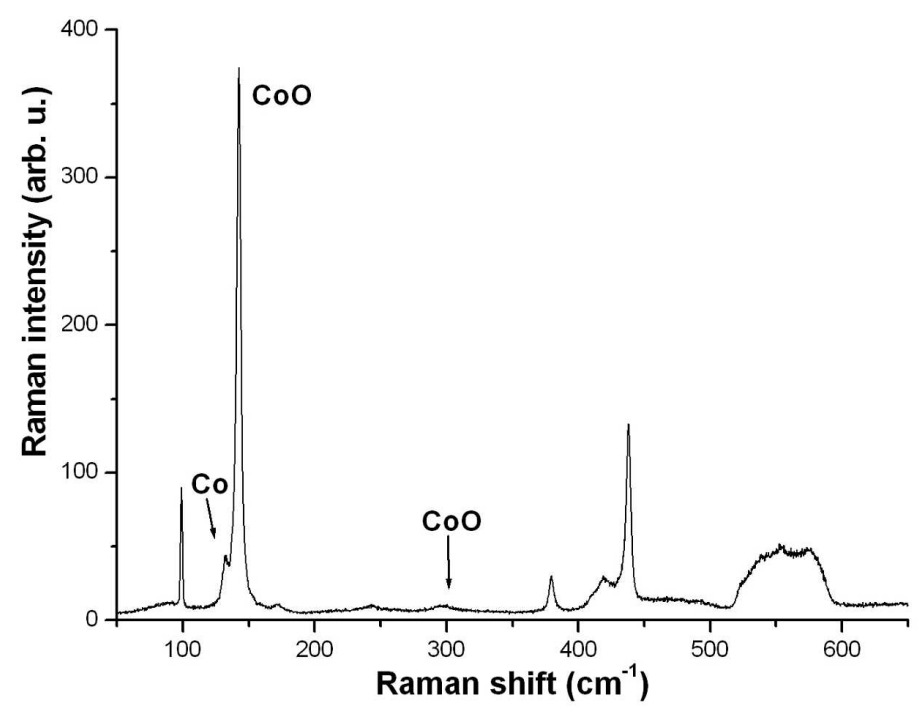

Fig. 1. Raman spectrum taken at $15 \mathrm{~K}$ with the $514.5 \mathrm{~nm} \mathrm{Ar}^{+}$laser line as the excitation source on a $\mathrm{ZnO}$ sample containing $1.6 \%$ of Co. The frequency positions of structures resulting from the presence of $\mathrm{Co}$ and $\mathrm{CoO}$ precipitates are indicated. For the discussion of the origin of the observed modes see the main text.

Some additional modes (or bands) in the spectral region between $500 \mathrm{~cm}^{-1}$ and $600 \mathrm{~cm}^{-1}$ are also well seen. Their presence was reported previously in the literature not only for $\mathrm{Zn}_{1-x} \mathrm{Co}_{x} \mathrm{O}$, but also for $\mathrm{ZnO}$ containing other dopants, like 
$\mathrm{Mn}$ or Ti. The mode with the maximum at about $539 \mathrm{~cm}^{-1}$ is due to the Co doping, so either it is disorder-induced or a Co-related mode. Another structure which could be related to Co LVM is a small peak at about $557 \mathrm{~cm}^{-1}$, reported previously in [16] (this structure does not appear both in pure $\mathrm{ZnO}$ and in $\mathrm{Zn}_{1-x} \mathrm{Mn}_{x} \mathrm{O}$ mixed crystals). Moreover, one can expect the presence of the $552 \mathrm{~cm}^{-1} B_{1}$ (high) normally silent mode, the activation of which has been predicted by theoretical calculations [17] as well as the $A_{1}(\mathrm{LO})$ and $E_{1}(\mathrm{LO})$ modes of pure $\mathrm{ZnO}$ which are situated in the spectral range $575-585 \mathrm{~cm}^{-1}$. All these modes may contribute to the total intensity of the broad band observed experimentally in the region of interest.

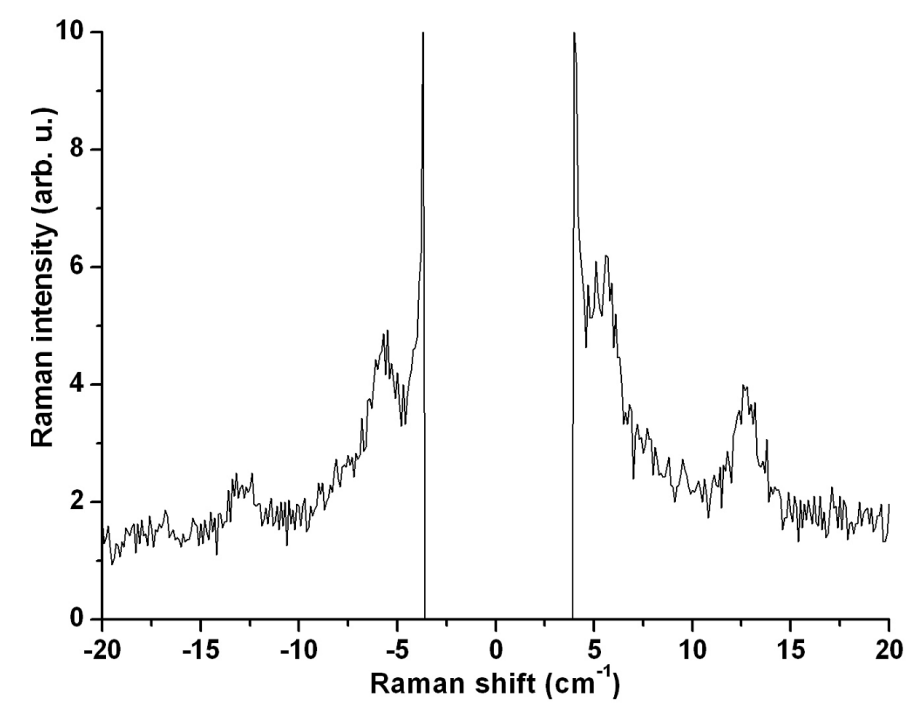

Fig. 2. Part of a Raman spectrum taken at the low frequency range on the same sample as that shown in Fig. 1. Both Stokes and anti-Stokes parts of the spectrum are shown. Sample temperature $T=15 \mathrm{~K}, \mathrm{Ar}^{+} 488 \mathrm{~nm}$ laser excitation line, laser power $100 \mathrm{~mW}$.

Figure 2 presents a low-frequency part of the Raman spectrum of the analyzed Co-doped $\mathrm{ZnO}$ sample measured with the use of the $488 \mathrm{~nm} \mathrm{Ar}^{+}$laser excitation line at $T=15 \mathrm{~K}$. One pair of structures $\left( \pm 5.4 \mathrm{~cm}^{-1}\right)$ shown in this figure corresponds to the splitting of the $\mathrm{Co}^{2+}$ ion ground state in the $\mathrm{ZnO}$ lattice due to the spin-orbit interaction and can be a direct confirmation of the local trigonal symmetry (an occupation of a substitutional site of the crystal lattice by an isolated $\mathrm{Co}^{2+}$ ion) [18]. The second pair of structures (about $\pm 13 \mathrm{~cm}^{-1}$ ) is probably due to an increase in the above-mentioned splitting for a part of $\mathrm{Co}^{2+}$ ions due to a deformation of the crystal lattice in the close neighborhood of these ions. Such a deformation may result from the possible presence of e.g., oxygen vacancies, zinc interstitials, or a second $\mathrm{Co}^{2+}$ ion. The same interpretation (creation of a complex containing $\mathrm{Co}^{2+}$ ion) can explain the lack of well-defined, sharp 
structures corresponding to a Co LVMs and the observation of the broad band between $500 \mathrm{~cm}^{-1}$ and $600 \mathrm{~cm}^{-1}$ (Fig. 1 and discussion above). On the other hand, the Raman scattering on the $\mathrm{Co}^{2+}$ ion ground state in the $\mathrm{CoO}$ lattice split due to the spin-orbit interaction is also possible [19] and such contribution to the total scattered intensity has to be taken into account. More detailed studies are clearly required in order to separate this contribution from the total signal. It should also be stressed that both pairs of structures shown in Fig. 2 are observed for the first time by the Raman scattering for Co-doped $\mathrm{ZnO}$ samples.

\section{Conclusions}

The analysis of the Raman spectra demonstrates that a big fraction of the $\mathrm{Co}^{2+}$ ions occupy the substitutional site of the cation sublattice. A part among them additionally forms a complex with e.g., oxygen vacancy, zinc interstitial, second $\mathrm{Co}^{2+}$ ion, etc. Moreover, the evidence of the presence of $\mathrm{Co}$ and $\mathrm{CoO}$ precipitates in a bulk crystal containing only $1.6 \%$ of Co was shown. This finding demonstrates the necessity of a careful structure analysis of materials from the TM-doped $\mathrm{ZnO}$ family considered as possible candidates for room temperature ferromagnetism. More experimental data are clearly required in order to get more detailed information on the nature of the above-mentioned complexes and about the local lattice deformation in $\mathrm{ZnO}$ with $\mathrm{Co}$.

\section{Acknowledgments}

The present work was supported in part by the POLONIUM project 7011/R07/R08.

\section{References}

[1] S.A. Wolf, D.D. Awschalom, Science 294, 1488 (2001).

[2] T. Dietl, H. Ohno, F. Matsukura, J. Cibert, D. Ferrand, Science 287, 1019 (2000).

[3] K. Sato, H. Katayama-Yoshida, Physica E 10, 251 (2001).

[4] Y. Wang, L. Sun, L.G. Kong, J.F. Kang, X. Zhang, R. Han, J. Alloys Comp. 423, $256(2006)$.

[5] A. Manivannan, P. Dutta, G. Glaspell, M.S. Seehra, J. Appl. Phys. 99, 08M110 (2006).

[6] G.L. Liu, Q. Cao, J.X. Deng, P.F. Xing, Y.F. Tian, Y.X. Chen, S.S. Yan, L.M. Mei, Appl. Phys. Lett. 90, 052504 (2007).

[7] M. Millot, J. Gonzalez, I. Molina, B. Salas, Z. Golacki, J.M. Broto, H. Rakoto, M. Goiran, J. Alloys Comp. 423, 224 (2006).

[8] K. Samanta, P. Bhattacharya, R.S. Katiyar, W. Iwamoto, P.G. Pagliuso, C. Rettori, Phys. Rev. B 73, 245213 (2006).

[9] P. Thurian, G. Kaczmarczyk, H. Siegle, R. Hertz, A. Hoffmann, I. Broser, B.K. Meyer, R. Hoffbauer, U. Scherz, Mater. Sci. Forum 196-201, 1571 (1995). 
[10] C. Schrepel, C. Göbel, U. Scherz, P. Thurian, G. Kaczmarczyk, A. Hoffmann, Z. Phys. Chem. 201, 21 (1997).

[11] W. Dobrowolski, M. Arciszewska, M. Klepka, A. Łusakowski, B. Brodowska, Z. Gołacki, unpublished.

[12] A.F. Goncharov, J. Crowhurst, J.M. Zaug, Phys. Rev. Lett. 92, 115502 (2004).

[13] R.R. Hayes, C.H. Perry, Solid State Commun. 13, 1915 (1973).

[14] H.-H. Chou, H.Y. Fan, Phys. Rev. B 13, 3924 (1976).

[15] X. Wang, J. Xu, B. Zhang, H. Yu, J. Wang, X. Zhang, J. Yu, Q. Li, Adv. Mater. 18, 2476 (2006).

[16] M. Jouanne, J.F. Morhange, W. Szuszkiewicz, Z. Gołacki, A. Mycielski, Phys. Status Solidi C 3, 1205 (2006).

[17] F.J. Manjon, B. Mari, J. Serrano, A.H. Romero, J. Appl. Phys. 97, 053516 (2005).

[18] P. Koidl, Phys. Rev. B 15, 2493 (1977).

[19] J. Sakurai, W.J.L. Buyers, R.A. Cowley, G. Dolling, Phys. Rev. 167, 510 (1968). 